\title{
Social Activity Networks Shaping St. Petersburg
}

\author{
David Landsman \\ Center for Urban Science and Progress, New York University, Brooklyn, NY, USA \\ MAP Centre for Urban Health Solutions, St. Michael's Hospital, Unity Health Toronto, Toronto, Canada \\ david.landsman@mail.utoronto.ca \\ Philipp Kats \\ Center for Urban Science and Progress, New York University, Brooklyn, NY, USA \\ Laboratory of Geophysics and Geoinformation Technologies, Institute of Geology and Petroleum Technologies, \\ Kazan Federal University, Kazan, Russia \\ casyfill@gmail.com \\ Aleksandra Nenko \\ ITMO University, Saint Petersburg, Russia \\ al.nenko@itmo.ru \\ Sergei Kudinov \\ Institute for Design and Urban Studies, ITMO University, Saint Petersburg, Russia \\ sergei.kudinov@itmo.ru \\ Stanislav Sobolevsky \\ Center for Urban Science and Progress, New York University, Brooklyn, NY, USA \\ ITMO University, Saint Petersburg, Russia \\ sobolevsky@nyu.edu
}

\begin{abstract}
Cities are complex systems, and understanding their structure is critical for multiple applications. However, traditional urban planning is challenged by the dynamics of the urban system. Fortunately, in recent years, multiple datasets reflecting human activity in nearly real-time have become available. This paper leverages geo-tagged data from VKontakte, Google Places social media and Nash Petersburg urban issue-reporting portal for building a multi-layered social activity network and revealing the structure of the city through the community structure in this network. The ability of this structure to capture meaningful socio-economic patterns across the city is evaluated. Results will aid urban, transportation, infrastructural planning, policy-making, real estate and socio-economic development initiatives.
\end{abstract}

\section{Introduction}

In recent years, data on human mobility and interactions in the city space saw an increasing number of applications. Data sources such as cell phone connections [1, 2, 3, 4, 5, 6, 7, 8], credit card transactions [9, 10, 11], GPS readings [12] as well as various sensor data [13, 14] were leveraged as proxies for human mobility. However, the availability of this data is often limited, largely due to privacy concerns [15. 2. 16]. On the other hand, social networks, location services, and municipal services provide a broader and more accessible alternative and have been proven useful for human mobility studies [17, 18, 19, 20, 21]. The spatial projection of the social network structure is known to reflect useful geographical information. In particular, these data sources have already been shown to allow for delineating regions at the country [1, 6, 7], global [17, 22], or local urban scales [4, 23]. A certain methodological challenge in using user-generated content as proxies for mobility is the natural bias in the social networks. Indeed, different social applications are used for different purposes and by different populations, so they are inherently biased by their nature. Moreover, urban mobility dynamics have an inherent complex nature, exhibiting various intentions. Thus, it could be impossible to fully understand it through any single data source, focusing on just one particular aspect of human behavior. Instead, using multiple sources together could reveal deeper 
aspects of spatial behavior, so that those sources would naturally complement each other. Overlapping geo-located data from different social media sources have already been successfully used in urban studies to cross-validate the city's non-administrative structure, places of interest, and mobility patterns [24, 25]. In addition, recent studies provided methodological background to deal with multi-layer complex networks [26, 22], allowing to build a single multilayered network combining different data sources. Using such a multi-layer network representing various aspects of human activity could provide a more complex, nuanced image than each data layer can provide individually [22].

In this study, we use four different sets of data, each representing different kinds of people's movements and interactions in the city of St. Petersburg, Russia, and contains: (1) user check-ins from Google places; (2) user check-ins from the Russian-language social network VKontakte (VK); (3) user friendship connections from VK; and (4) non-emergency issues tracked by "Nash Petersburg" municipal service; All data except (4) is collected from July 2017 up to July 2018. Nash Petersburg dataset fully covers the years 2017 and 2018.

Although Google and VK datasets are similar and both originate from social media and contain geographical and social data, they still represent different types of people's activity and different user populations. Namely, VK mostly reflects the social aspect of mobility, for example, attendance of events or specific venues, while Google has a more diverse representation of venue types. The VK network of friendships, in its turn, represents long-term social interactions in-between the users. Google Places data has proved itself to be illustrative for urban structure analysis, in particular, analysis of urban neighborhood regeneration [25] and land use [27]. VK is the most popular social network in Russia. Due to its representativeness and availability of the check-in data and data on social ties, it is applicable for analyzing everyday mobility and urban space usage [28, 29]. On the other hand, "Nash Petersburg" represents people's day-to-day concerns and, as such, could be more representative of daily mobility and areas closer to people's homes. Indeed, using data from similar sources (namely, 311) in the US has shown the significance and value of this data for urban modeling [30, 31, 32]. When performing our analyses, we consider network nodes to represent the city's administrative divisions: city districts and municipalities.

By redefining those datasets as networks of locations, using agents (people or venues) to establish connections between them, we are able to provide a quantitative representation for different aspects of human mobility. Our study's specific focus is demonstrating that such data sources could provide valuable insights on urban structure and support decision making. For that purpose, we apply community detection to the individual networks, as well as to the multi-layer network combining all four networks together. The resulting community structures are compared against each other and evaluated against the existing administrative partition of the city. They are further analyzed with respect to the socio-economic statistics of the city, including census demographics and real estate characteristics. The ability of the resulting network structure to reveal meaningful socio-economic patterns across the city is also evaluated.

\section{Methods}

\subsection{Data and Networks}

The data for social network analysis spanning from 2010 to 2018 was extracted in July 2018 from Google ${ }^{1}$ and $\mathrm{VK}^{2}$ Data on Google and VK places contains a unique identifier, address, name, latitude and longitude for each venue. This data also contains a unique identifier for each user, and data about user check-ins, which links users to places they visited via the user and place identifiers. VK data additionally contains friendship connections between users. Nash Petersburg dat: ${ }^{3}$ covers two full years of 2017-2018 and contains latitude, longitude, type of issue, time of creation, and anonymized ID of the author. Table 1 presents a summary of the data.

Table 1: Data summary.

\begin{tabular}{|llll|}
\hline & Google & VKontakte & $\begin{array}{l}\text { Nash } \\
\text { Petersburg }\end{array}$ \\
\hline \hline $\begin{array}{l}\text { Unique } \\
\text { places }\end{array}$ & 63,391 & 62,906 & N/A \\
\hline $\begin{array}{l}\text { Unique } \\
\text { users }\end{array}$ & 993,379 & 268,297 & 46,449 \\
\hline $\begin{array}{l}\text { Unique } \\
\text { check-ins }\end{array}$ & $2,824,832$ & 765,133 & $1,214,225$ \\
\hline Date range & $2010-2018$ & $2010-2018$ & $2017-2018$ \\
\hline
\end{tabular}

The data used for the evaluation of the network analysis consists of: (1) demographic data at the district-level of St. Petersburg, including social groups

\footnotetext{
https://www.google.com/maps/

https://vk.com/

https://gorod.gov.spb.ru/
} 
stratified by age, gender, and education level retrieved from the city official statistical porta 4 , (2) building characteristics, including addresses and repair details 5 . and (3) real estate data, including price, amenities and addresses of building 6

2.1.1. Google An initial place similarity network was constructed from the Google Places data by representing each place with a node, and connecting two nodes with a weighted undirected edge representing the number of users who have ever visited both places. This approach for network construction is similar to the approaches used for mobility networks such as [17. 22]. The place similarity network was then converted into a municipality similarity network and a district similarity network by mapping each place's latitude and longitude to the corresponding municipality and district, respectively. Weights of edges connecting between municipalities (respectively, districts) are computed by summing the weights of edges between places in the corresponding municipalities (respectively, districts) together.

2.1.2. VKontakte Using the raw data extracted from VK, we constructed two initial networks: a place similarity network similar to the Google network in Section 2.1.1 and a friendship network. The friendship network was constructed by representing each user with a node, and each friendship relationship with an edge of weight one. We then constructed municipality similarity networks and district similarity networks similarly to the method described in Section 2.1.1. To map each user in the friendship network to a corresponding municipality (respectively, district), we selected the most frequent municipality (respectively, district) out of the user's check-ins, breaking ties by choosing the municipality (respectively, district) with the higher identifier. This method is similar to the approaches for defining a user's home location analyzed in [33].

The resulting municipality similarity networks constructed from VK place similarity and friend networks contained many municipalities with sparse data, resulting in scattered clusters. To overcome this issue, we included only those nodes which attained at least $1 \%$ of the maximum node strength in the network; that is, only nodes $u$ which satisfy the equation:

$$
\frac{\sum_{v \in \mathcal{V}} w(u, v)}{\max \left\{\sum_{v \in \mathcal{V}} w\left(u^{\prime}, v\right) \mid u^{\prime} \in \mathcal{V}\right\}} \geq 0.01
$$

where $w(u, v)$ is the weight of edge $(u, v)$ of the network $G=(\mathcal{V}, \mathcal{E})$.

2.1.3. Nash Petersburg Using Nash Petersburg data, we first constructed a partition, based on the normalized distributions of issues by geographical area and issue type, similar to [30]. We used the $k$-Means algorithm together with the Silhouette method to define the number of clusters $(k=5)$. While this approach performs reasonably well on the district level (Figure 1 ), it is not of particular use on the municipality level due to the small number of records for municipalities located farther from the city center. To mitigate this, we constructed municipal and district-level user mobility network, similarly to Google places in Section 2.1.1

However, the number of issues reported by each user varies significantly. Due to this heterogeneity in user activity, as well as the comparatively small number of users, we chose to weigh the network edges based on the user's distribution of records, similar to the method in [21]. For this network, nodes represent municipalities (respectively, districts), and edge weights are computed as a sum of products of activity ratios between each pair of two areas by the given user:

$$
w(A, B)=\sum_{u} \frac{V(u, A)}{\sum_{C} V(u, C)} \frac{V(u, B)}{\sum_{C} V(u, C)}
$$

where $w(A, B)$ is the weight of edge $(A, B)$ of the network $G=(\mathcal{V}, \mathcal{E})$, and $V(u, A)$ is number of issues raised by user $u$ in area $A$.

To further reduce the noise, we only include accounts with at least 5 issues, which account for approximately $95.2 \%$ of all issues.

\subsection{Network Clustering}

We investigated the clustering of the networks using the community detection algorithm Combo [34]. The Combo algorithm optimizes the modularity function [35] by splitting, merging, and recombining subsets of nodes into clusters. Combo was chosen for its great performance in maximizing modularity when compared to state of the art algorithms such as simulated annealing and the Louvain method, as well as its adequate runtime

${ }^{4}$ https://petrostat.gks.ru/

5 http://data.gov.spb.ru/opendata/7840013199-passports_houses/versions/ 'https://spb.cian.ru/ for the types of networks we are considering in this paper [34]. 
In the original definition of modularity, we compare the number of edges placed into clusters with the expected number of edges per cluster based on a random repositioning of the edges in the network. It can be computed as:

$$
\frac{1}{2 m} \sum_{i \neq j}\left(A_{i j}-\frac{k_{i} k_{j}}{2 m}\right) \delta\left(g_{i}, g_{j}\right)
$$

where $m$ is the total number of edges in the network, $A_{i j}$ is the weight of edge $(i, j), k_{i}:=\sum_{j} A_{i j}$ is the node strength of node $i, g_{i}$ is the cluster of node $i$ and $\delta(x, y)=1$ if and only if $x=y$.

It has been shown that the classical definition of modularity suffers from a resolution limit [36, 37], preventing it from finding small clusters in large networks. [36] proposes a modification of modularity by introducing a real-valued resolution hyperparameter $\gamma$ :

$$
Q=\frac{1}{2 m} \sum_{i \neq j}\left(A_{i j}-\gamma \frac{k_{i} k_{j}}{2 m}\right) \delta\left(g_{i}, g_{j}\right)
$$

We also investigated the clustering of the multi-layer network constructed by combining the (1) Google place-similarity network; (2) VK place-similarity network; (3) VK friendship network; and (4) Nash Petersburg user mobility network. As before, each node in the multi-layer network represents a municipality or a district of St. Petersburg, however, there may be multiple edges between the same nodes, corresponding to the different layers of the network. In order to apply the Combo algorithm to the multi-layer network we followed the work in [22] to formulate a multi-layer version of modularity:

$$
Q^{\prime}=\frac{1}{4} \sum_{l=1}^{4} \alpha_{l} Q_{l}
$$

where $l$ denotes the network layer, $\alpha_{l}$ is the weight applied to layer $l$, and $Q_{l}$ is the modularity of layer $l$ as computed in Equation (4).

\subsection{Evaluation}

In order to evaluate and optimize the choice of the resolution hyperparameter $\gamma$ in Equation (4) and the modularity weights $\alpha_{l}$ in Equation (5) when clustering the municipality networks, we computed the normalized mutual information (NMI) score between the true district structure of St. Petersburg and the resulting clusters. Given two partitions $\mathcal{A}$ and $\mathcal{B}$ of $\mathcal{V}$ into disjoint subsets of nodes, let $C$ be the confusion matrix of size $|\mathcal{A}| \times|\mathcal{B}|$ with $C_{i j}$ indicating the number of nodes belonging to cluster $i$ of partition $\mathcal{A}$ and cluster $j$ of partition $\mathcal{B}$. Then, NMI score can be computed as following:

$$
\begin{aligned}
& \operatorname{NMI}(\mathcal{A}, \mathcal{B})= \\
& \frac{-2 \sum_{i=1}^{|\mathcal{A}|} \sum_{j=1}^{|\mathcal{B}|} C_{i j} \log \left(\frac{C_{i j}|\mathcal{V}|}{C_{i \cdot} \cdot C_{\cdot j}}\right)}{\sum_{i=1}^{|\mathcal{A}|} C_{i} \cdot \log \left(\frac{C_{i \cdot}}{|\mathcal{V}|}\right)+\sum_{j=1}^{|\mathcal{B}|} C_{\cdot j} \log \left(\frac{C_{\cdot j}}{|\mathcal{V}|}\right)}
\end{aligned}
$$

However, as noted in [38] using NMI score in the optimization of community detection can lead to a high number of clusters being produced. Instead, the authors suggest to use a fair normalized mutual information (FNMI) score which penalizes cluster structures that differ too greatly from the true delineation:

$$
\begin{aligned}
& \operatorname{FNMI}(\mathcal{A}, \mathcal{B})= \\
& \qquad \exp \left\{\frac{-|| \mathcal{A}|-| \mathcal{B}||}{|\mathcal{A}|}\right\} \cdot \operatorname{NMI}(\mathcal{A}, \mathcal{B})
\end{aligned}
$$

Note that in clustering the district-level networks, we set the resolution hyperparameter $\gamma=1$ and the multi-layer weights to be $\alpha_{l}=1$ for all four networks as there is no ground-truth partition to fit.

We evaluated the performance of the district-level clustering in modeling various municipalityand district-level outcome variables from our socio-economic datasets. We performed a weighted least squares regression for each cluster structure (multi-layer network, Google place-similarity network, VK place-similarity network, VK friendship network, Nash Petersburg mobility network) using the cluster identifier as a categorical variable, and setting weights equal to the inverse of municipality and district populations. The outcome variables we considered in our analysis are presented in Table 2 . Note that some of the variables were only available on the district-level.

\section{Results}

\subsection{Network Clustering}

Figure 1 presents the clustering of the multi-layer, Google, VK and Nash Petersburg district similarity networks using Combo. Figure 2 presents the clustering of the multi-layer, Google, VK and Nash Petersburg municipality similarity networks. 


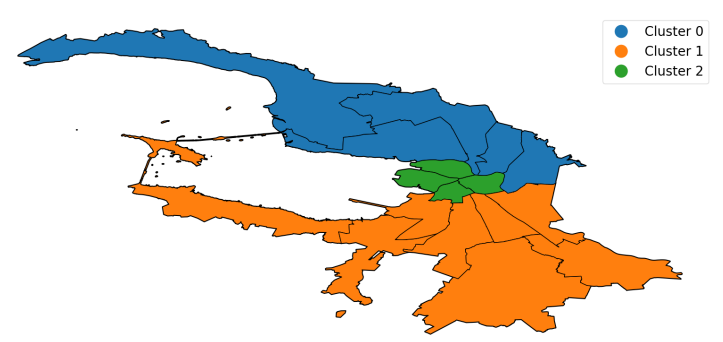

(a) Multi-layer network

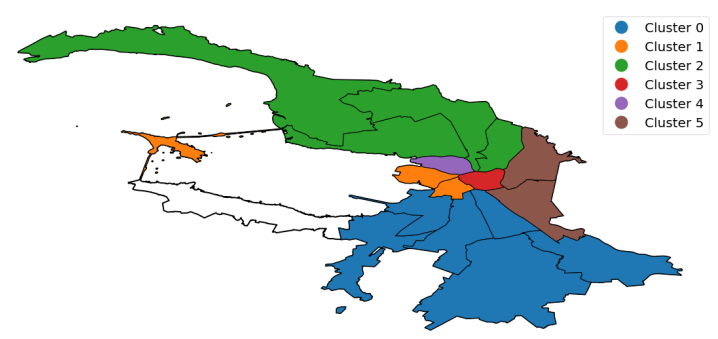

(c) VK place-similarity network

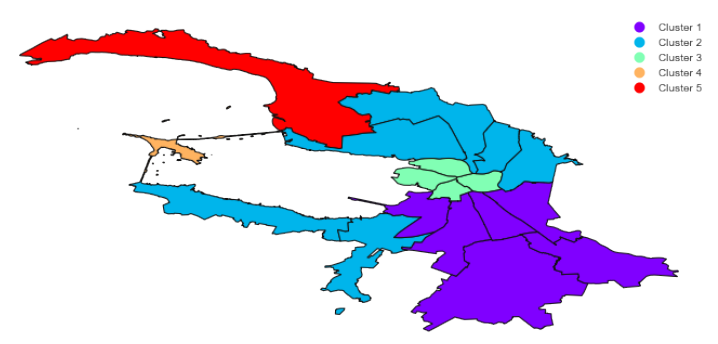

(e) Nash Petersburg issue type-based clustering

*Blank regions indicate districts that were not included in the networks due to limited data.

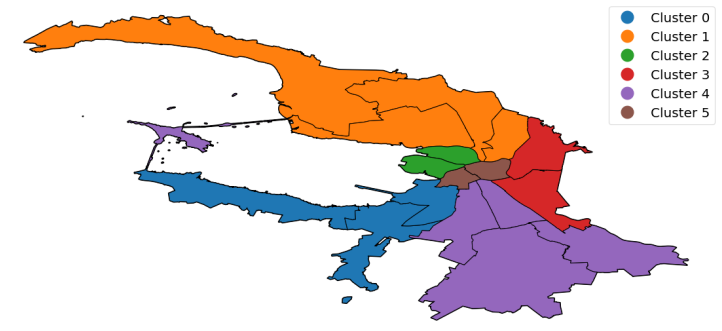

(b) Google place-similarity network

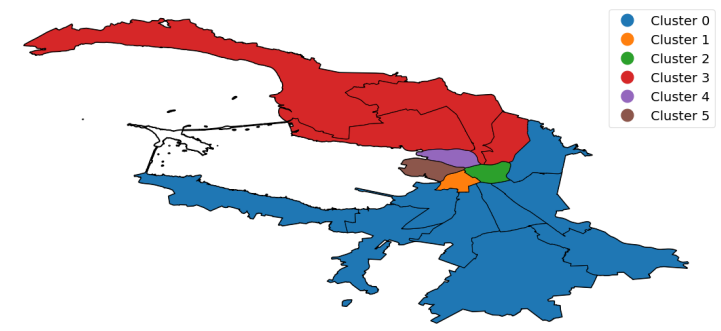

(d) VK friendship network

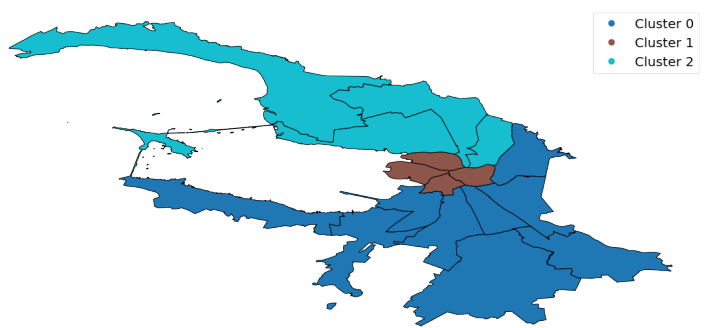

(f) Nash Petersburg mobility network

Figure 1: Clustering of district-level networks of St. Petersburg.

Table 2: Outcome variables for regression analysis on district-level clustering.

\begin{tabular}{|lll|}
\hline Variable Name & Granularity & Source \\
\hline \hline $\begin{array}{l}\text { Middle-aged workes } \\
\text { (rate per 10,000) }\end{array}$ & District & Demographics \\
\hline $\begin{array}{l}\text { Pre-school children } \\
\text { (rate per 10,000) }\end{array}$ & District & Demographics \\
\hline $\begin{array}{l}\text { School-age children } \\
\text { (rate per 10,000) }\end{array}$ & District & Demographics \\
\hline $\begin{array}{l}\text { Median house prices } \\
\text { in rubles (log-scale) }\end{array}$ & Municipality & Real estate \\
\hline $\begin{array}{l}\text { Median house sizes } \\
\text { in square meters } \\
\text { (log-scale) }\end{array}$ & Municipality & Real estate \\
\hline $\begin{array}{l}\text { Buildings per } \\
\text { square kilometer }\end{array}$ & Municipality & $\begin{array}{l}\text { Building } \\
\text { characteristics }\end{array}$ \\
\hline
\end{tabular}

In the district clustering, we see a clear division of the city into Northern, Southern and central regions. This division corresponds the vernacular division of the city. It also aligns with the structure of the subway network which has the most developed branches heading South and North of the city. The amenities and residential housing in St. Petersburg tend to concentrate in proximity to subway stations, consequently patterns of place check-ins resemble its structure. There are noticeable variations among the networks in the clustering of Eastern and central districts. For the central district it is most possibly connected with the diversity of this area being a distinctive leisure and socializing place and, at the same time, a residential territory. These uses are captured differently in Google, VK and Nash Petersburg data. The multi-layer network partition seems to stay clear of the discrepancies between individual network partitions and focus on the most significant spatial patterns, clearly delineating the center, North and South regions. 


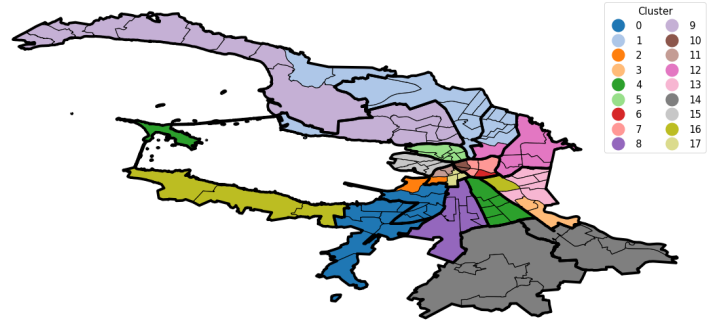

(a) Multi-layer network

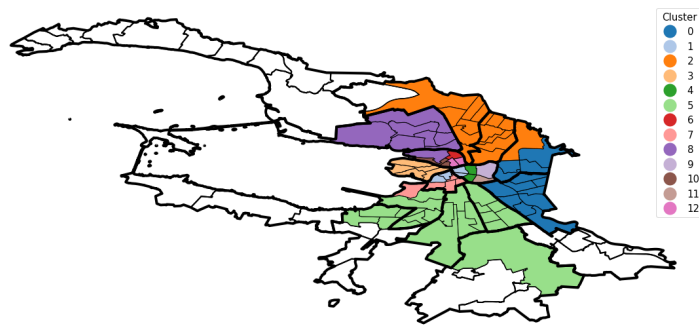

(c) VK place-similarity network*

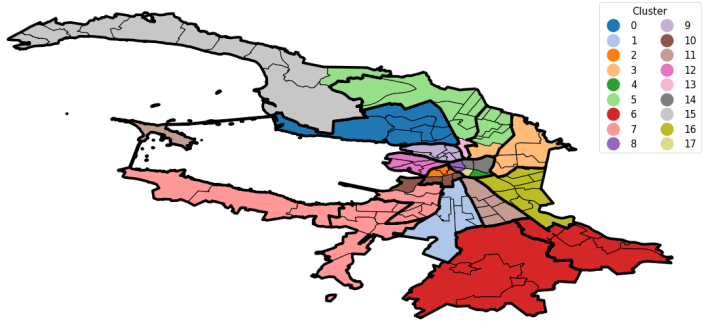

(b) Google place-similarity network

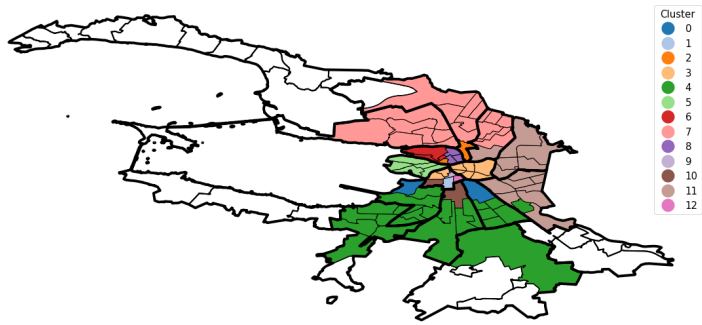

(d) VK friendship network*

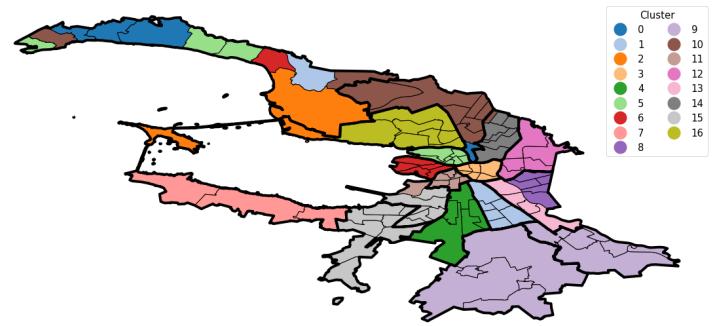

(e) Nash Petersburg mobility network

*Blank regions indicate districts that were not included in the networks due to limited data.

Figure 2: Clustering of municipality-level networks of St. Petersburg. True district borders highlighted in bold.

For the municipality clustering we see a much wider range of results. Aligned with our optimization objective, some of the municipality clusters resemble the true district structure of St. Petersburg, especially when the data is more abundant as in the Google place-similarity or the Nash Petersburg mobility networks. The multi-layer network is also able to capture much of the true district structure, due to the contributions of both Google and Nash Petersburg networks. However, due to scarcity of municipality-level activity in the VK networks and the related noisiness, the clusters for these networks are less aligned with the districts.

Table 3 presents the FNMI scores for the optimal clustering of Google and VK municipality-level data in comparison with the true district structure of St. Petersburg. As could be seen in Figure 2, the Google, Nash Petersburg and multi-layer networks achieve higher FNMI scores than the VK networks.
Table 3: Fair normalized mutual information (FNMI) score of Combo clusters created from municipality-level networks, and true district structure of St. Petersburg.

\begin{tabular}{|ll|}
\hline Partition & FNMI \\
\hline \hline Multi-layer network & 0.8518 \\
\hline Google place-similarity network & 0.8868 \\
\hline VK place-similarity network & 0.7199 \\
\hline VK friendship network & 0.6656 \\
\hline Nash Petersburg mobility network & 0.8409 \\
\hline
\end{tabular}

\subsection{Socio-Economic Analysis of Clusters}

Figure 3 shows the adjusted $R^{2}$ values computed from the regressions of the socio-economic characteristics of St. Petersburg districts on the cluster structure (using cluster attachment binary variables as regressors). Overall we see reasonable performance across the majority of the networks, with 


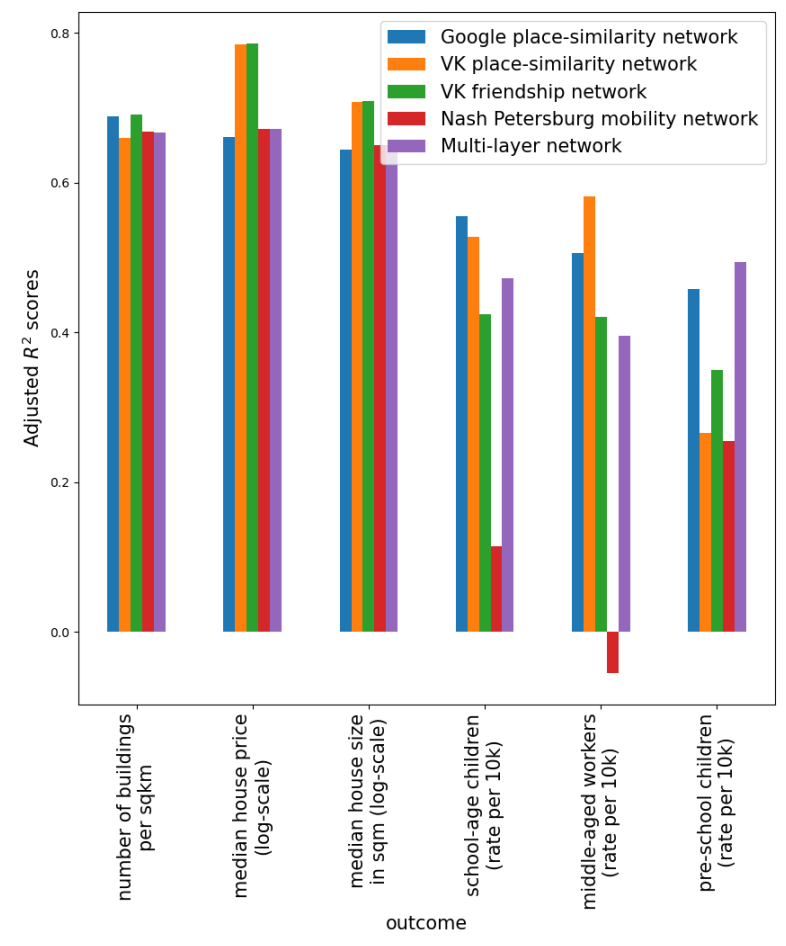

Figure 3: Comparison of adjusted $R^{2}$ scores from regression of socio-economic outcomes across multi-layer, Google, VK places, VK friends and Nash Petersburg district-level cluster structures.

somewhat weaker performance of the Nash Petersburg network in the demographic outcomes. This highlights the utility of the resulting network structure in capturing meaningful socio-economic patterns across the city.

Figure 4 shows the socio-economic profile of the clusters as represented by the regression coefficients. All the networks appear to cluster the city into zones of distinctive socio-economic profile. Notably, VK places and friendship networks have slightly better coverage of real-estate and building density patterns than the rest of the networks.

\section{Discussion and Conclusions}

We see three different types of people's movements and interactions - user check-ins on Google Places and VKontakte; friendships in VKontakte; and mobility network based on Nash Petersburg provide different but largely consistent perspectives on urban zoning. Cluster structures from all four delineate the city center and major areas on the district map and provide municipality clustering consistent with the city's district borders. Moreover, the joint multi-layer network clustering offers the clearest delineation of the city center, Northern and
Southern regions.

The clustering of the municipality-level networks corresponds to the vernacular division of St. Petersburg into the diverse historic center, which is presented by at least two clusters in each network (except Nash Petersburg), which mostly illustrates people's commute patterns and the large Northern and Southern regions. Less distinctive Eastern and Western regions are captured only with Google Places data. The similarity of the obtained clustering and the administrative and vernacular divisions is greatest for the Google Places data, the most abundant source of information about venues used daily. However, it is also a point for future elaboration on the limitations of different datasets. In particular, VK places similarity network is less illustrative for the patterns of using venues in the city, as VK's key function is not an aggregation of places, but social networking.

The provided urban zoning turns out to be highly consistent with real-estate patterns, as well as highlighting meaningful socio-economic patterns. For all types of clustering, knowing which cluster the district belongs to allows to explain up to $70 \%$ of the variance in building density, nearly $80 \%$ in real-estate prices, and around $50-60 \%$ of the variance in demographics decomposition. However, different networks highlight slightly distinctive patterns: e.g., VK friendship shows a stronger relationship with the real estate characteristics, VK place-similarity with the working population, Google places with school-aged children, while multi-layer network - with pre-school-aged children.

The differences in clustering results can highlight meaningful socio-economic patterns. Using multiple data sets to approach the zoning problem provides some complementarity, especially given the data quality and other limitations of each particular data set. Bringing multiple data layers together within the multi-layered network offers additional benefits evident in a robust cluster structure. Therefore, including additional data sets on various aspects of social activity could be beneficial. The results could benefit urban and transportation planners, policymakers, developers, urban planners, and other urban stakeholders.

\section{Acknowledgements}

The authors thank Artem Koniukhov and Maria Podkorytova for their valuable help with the data acquisition and processing. We further thank Sergey Mityagin for supporting this research collaboration. This work was financially supported by the National Center for Cognitive Research of ITMO University. 


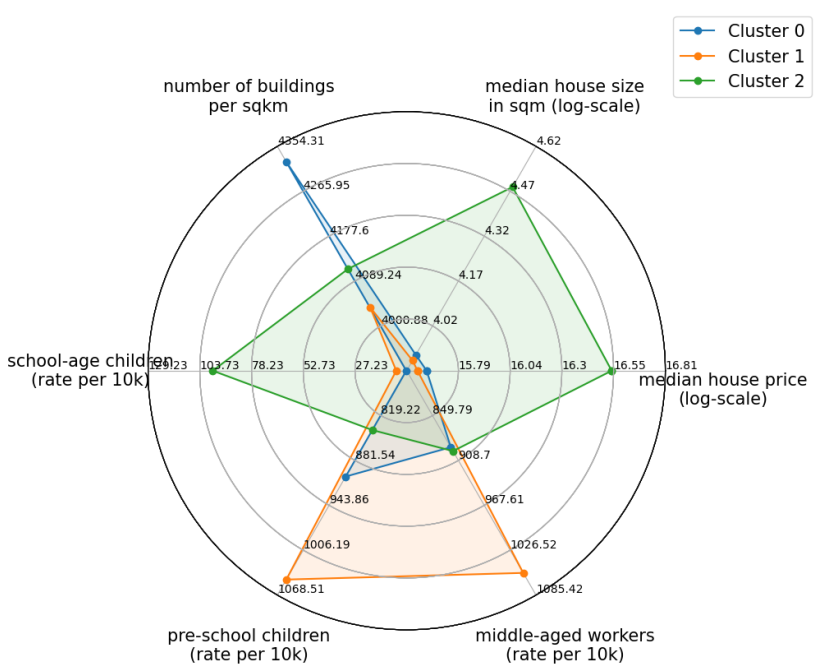

(a) Multi-layer network

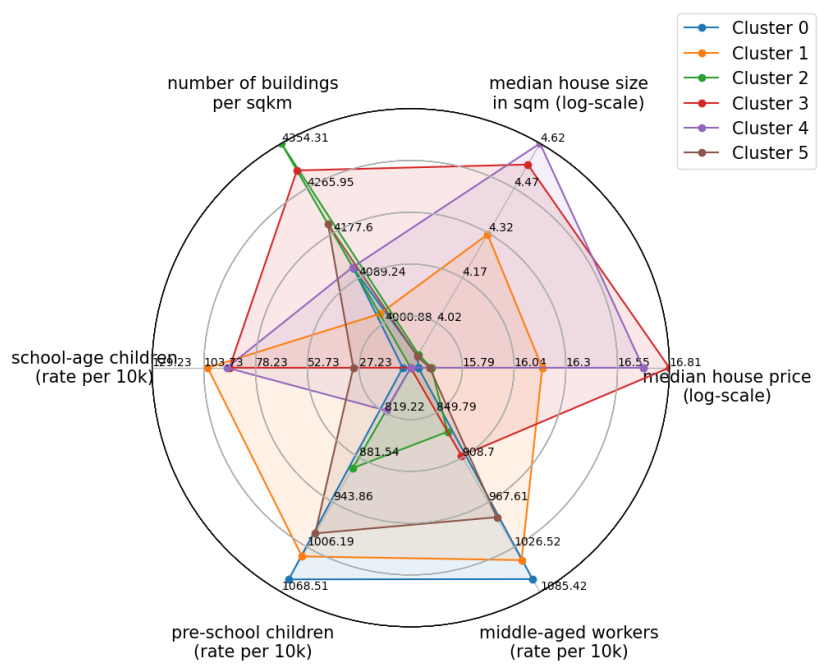

(c) VK place-similarity network

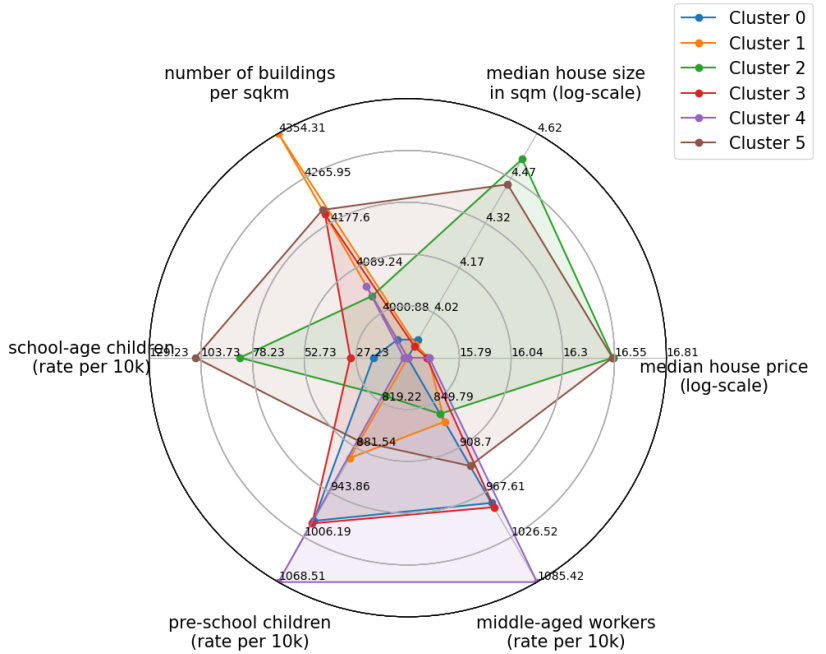

(b) Google place-similarity network

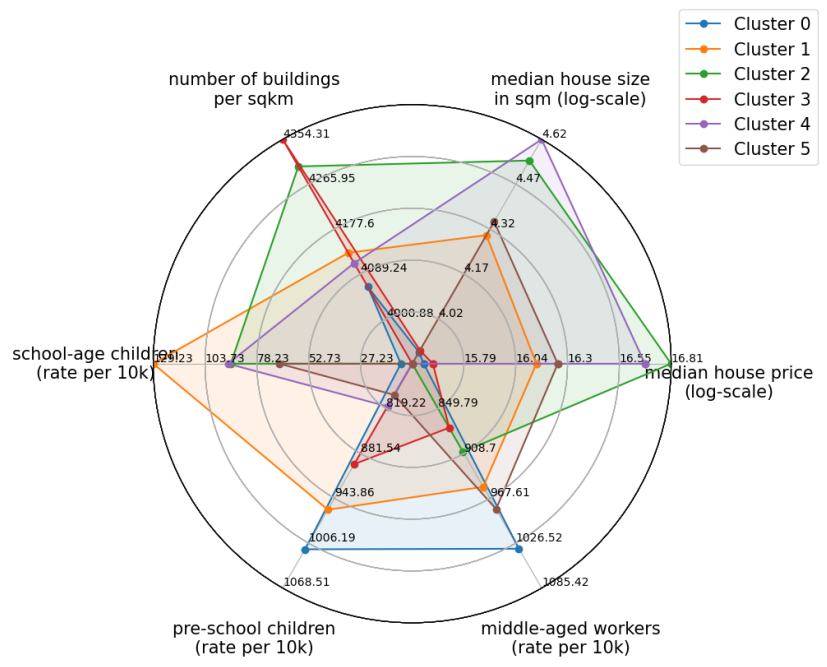

(d) VK friendship network

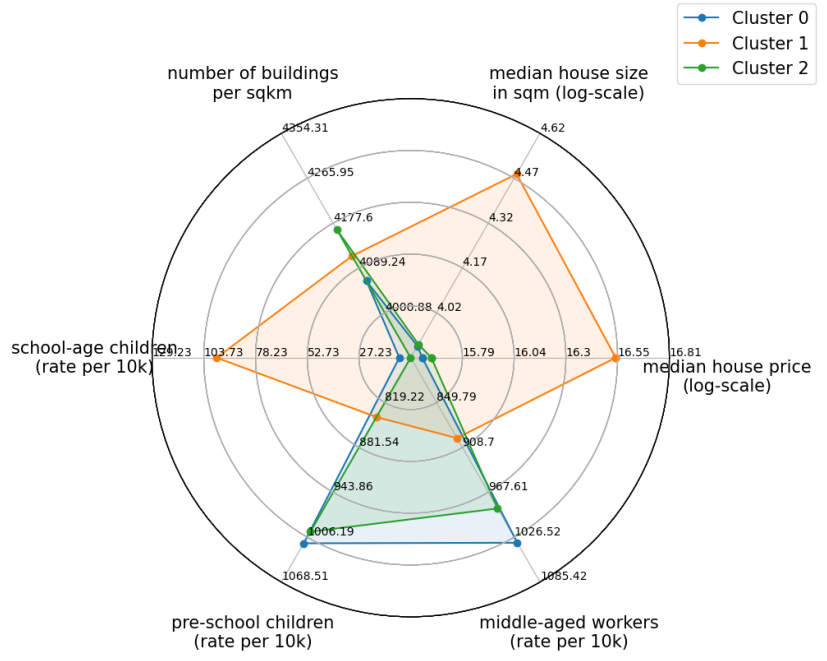

(e) Nash Petersburg mobility network

Figure 4: Comparison of socio-economic outcomes across multi-layer, Google, VK places, VK friends and Nash Petersburg district-level cluster structures. 


\section{References}

[1] A. Amini, K. Kung, C. Kang, S. Sobolevsky, and C. Ratti, "The impact of social segregation on human mobility in developing and industrialized regions," $E P J$ Data Science, vol. 3, p. 6, June 2014.

[2] F. Girardin, F. Calabrese, F. D. Fiore, C. Ratti, and J. Blat, "Digital Footprinting: Uncovering Tourists with User-Generated Content," IEEE Pervasive Computing, vol. 7, pp. 36-43, Oct. 2008. Conference Name: IEEE Pervasive Computing.

[3] M. C. González, C. A. Hidalgo, and A.-L. Barabási, "Understanding individual human mobility patterns," Nature, vol. 453, pp. 779-782, June 2008. Number: 7196 Publisher: Nature Publishing Group.

[4] C. Kang, S. Sobolevsky, Y. Liu, and C. Ratti, "Exploring human movements in Singapore: a comparative analysis based on mobile phone and taxicab usages," in Proceedings of the 2nd ACM SIGKDD International Workshop on Urban Computing, UrbComp '13, (Chicago, Illinois), pp. 1-8, Association for Computing Machinery, Aug. 2013.

[5] D. Quercia, N. Lathia, F. Calabrese, G. Di Lorenzo, and J. Crowcroft, "Recommending Social Events from Mobile Phone Location Data," in 2010 IEEE International Conference on Data Mining, pp. 971-976, Dec. 2010. ISSN: 2374-8486.

[6] C. Ratti, S. Sobolevsky, F. Calabrese, C. Andris, J. Reades, M. Martino, R. Claxton, and S. H. Strogatz, "Redrawing the Map of Great Britain from a Network of Human Interactions," PLOS ONE, vol. 5, p. e14248, Dec. 2010. Publisher: Public Library of Science.

[7] S. Sobolevsky, M. Szell, R. Campari, T. Couronné, Z. Smoreda, and C. Ratti, "Delineating Geographical Regions with Networks of Human Interactions in an Extensive Set of Countries," PLOS ONE, vol. 8, p. e81707, Dec. 2013. Publisher: Public Library of Science.

[8] J. Reades, F. Calabrese, A. Sevtsuk, and C. Ratti, "Cellular Census: Explorations in Urban Data Collection," IEEE Pervasive Computing, vol. 6, pp. 30-38, July 2007. Conference Name: IEEE Pervasive Computing.

[9] S. Sobolevsky, I. Bojic, A. Belyi, I. Sitko, B. Hawelka, J. M. Arias, and C. Ratti, "Scaling of City Attractiveness for Foreign Visitors through Big Data of Human Economical and Social Media Activity," in 2015 IEEE International Congress on Big Data, pp. 600-607, June 2015. ISSN: 2379-7703.

[10] S. Sobolevsky, I. Sitko, R. T. D. Combes, B. Hawelka, J. M. Arias, and C. Ratti, "Money on the Move: Big Data of Bank Card Transactions as the New Proxy for Human Mobility Patterns and Regional Delineation. The Case of Residents and Foreign Visitors in Spain," in 2014 IEEE International Congress on Big Data, pp. 136-143, June 2014. ISSN: 2379-7703.

[11] S. Sobolevsky, I. Sitko, R. T. d. Combes, B. Hawelka, J. M. Arias, and C. Ratti, "Cities through the Prism of People's Spending Behavior," PLOS ONE, vol. 11, p. e0146291, Feb. 2016. Publisher: Public Library of Science.

[12] P. Santi, G. Resta, M. Szell, S. Sobolevsky, S. H. Strogatz, and C. Ratti, "Quantifying the benefits of vehicle pooling with shareability networks," Proceedings of the National Academy of Sciences, vol. 111, pp. 13290-13294, Sept. 2014.
[13] N. Lathia, D. Quercia, and J. Crowcroft, "The Hidden Image of the City: Sensing Community Well-Being from Urban Mobility," in Pervasive Computing (J. Kay, P. Lukowicz, H. Tokuda, P. Olivier, and A. Krüger, eds.), Lecture Notes in Computer Science, (Berlin, Heidelberg), pp. 91-98, Springer, 2012.

[14] C. E. Kontokosta and N. Johnson, "Urban phenology: Toward a real-time census of the city using Wi-Fi data," Computers, Environment and Urban Systems, vol. 64, pp. 144-153, July 2017.

[15] F. Bélanger and R. E. Crossler, "Privacy in the Digital Age: A Review of Information Privacy Research in Information Systems," MIS Quarterly, vol. 35, no. 4, pp. 1017-1041, 2011. Publisher: Management Information Systems Research Center, University of Minnesota.

[16] J. Lane, V. Stodden, S. Bender, and H. Nissenbaum, Privacy, big data, and the public good: Frameworks for engagement. Cambridge University Press, Jan. 2013.

[17] B. Hawelka, I. Sitko, E. Beinat, S. Sobolevsky, P. Kazakopoulos, and C. Ratti, "Geo-located Twitter as proxy for global mobility patterns," Cartography and Geographic Information Science, vol. 41, pp. 260-271, May 2014. Publisher: Taylor \& Francis _eprint: https://doi.org/10.1080/15230406.2014.890072.

[18] A. Kurkcu, E. Morgul, and K. Ozbay, "Evaluating the Usability of Geo-Located Twitter as a Tool for Human Activity and Mobility Patterns: A Case Study for New York City," Jan. 2016.

[19] S. Paldino, I. Bojic, S. Sobolevsky, C. Ratti, and M. C. González, "Urban magnetism through the lens of geo-tagged photography," EPJ Data Science, vol. 4, p. 5, May 2015.

[20] C. Qian, P. Kats, S. Malinchik, M. Hoffman, B. Kettler, C. Kontokosta, and S. Sobolevsky, "Geo-Tagged Social Media Data as a Proxy for Urban Mobility," in Advances in Cross-Cultural Decision Making (M. Hoffman, ed.), Advances in Intelligent Systems and Computing, (Cham), pp. 29-40, Springer International Publishing, 2018.

[21] S. Sobolevsky, P. Kats, S. Malinchik, M. Hoffman, B. Kettler, and C. Kontokosta, "Twitter Connections Shaping New York City," Jan. 2018. Accepted: 2017-12-28T00:43:38Z.

[22] A. Belyi, I. Bojic, S. Sobolevsky, I. Sitko, B. Hawelka, L. Rudikova, A. Kurbatski, and C. Ratti, "Global multi-layer network of human mobility," International Journal of Geographical Information Science, vol. 31, pp. 1381-1402, July 2017. Publisher: Taylor \& Francis _eprint: https://doi.org/10.1080/13658816.2017.1301455.

[23] T. Louail, M. Lenormand, O. G. Cantu Ros, M. Picornell, R. Herranz, E. Frias-Martinez, J. J. Ramasco, and M. Barthelemy, "From mobile phone data to the spatial structure of cities," Scientific Reports, vol. 4, p. 5276, June 2014. Number: 1 Publisher: Nature Publishing Group.

[24] T. Agryzkov, P. Martí, A. Nolasco-Cirugeda, L. Serrano-Estrada, L. Tortosa, and J. F. Vicent, "Analysing successful public spaces in an urban street network using data from the social networks foursquare and twitter," Applied network science, vol. 1, no. 1, p. 12, 2016.

[25] P. Martí, C. García-Mayor, and L. Serrano-Estrada, "Identifying opportunity places for urban regeneration through lbsns," Cities, vol. 90, pp. 191-206, 2019. 
[26] M. Kivelä, A. Arenas, M. Barthelemy, J. P. Gleeson, Y. Moreno, and M. A. Porter, "Multilayer networks," Journal of Complex Networks, vol. 2, pp. 203-271, Sept. 2014. Publisher: Oxford Academic.

[27] S. Jiang, A. Alves, F. Rodrigues, J. Ferreira Jr, and F. C. Pereira, "Mining point-of-interest data from social networks for urban land use classification and disaggregation," Computers, Environment and Urban Systems, vol. 53, pp. 36-46, 2015.

[28] A. Zagarskikh, A. Karsakov, and T. Tchurov, "The framework for problem solving environments in urban science," Procedia Computer Science, vol. 29, pp. 2483-2495, 2014.

[29] A. Nenko, A. Koniukhov, and M. Petrova, "Areas of habitation in the city: improving urban management based on check-in data and mental mapping," in International Conference on Electronic Governance and Open Society: Challenges in Eurasia, pp. 235-248, Springer, 2018.

[30] L. Wang, C. Qian, P. Kats, C. Kontokosta, and S. Sobolevsky, "Structure of 311 service requests as a signature of urban location," vol. 12, no. 10, p. e0186314. Publisher: Public Library of Science.

[31] P. Kats, C. Qian, C. Kontokosta, and S. Sobolevsky, "Twitter activity timeline as a signature of urban neighborhood,"

[32] E. Zhu, M. Khan, P. Kats, S. S. Bamne, and S. Sobolevsky, "Digital urban sensing: A multi-layered approach,"

[33] I. Bojic, E. Massaro, A. Belyi, S. Sobolevsky, and C. Ratti, "Choosing the right home location definition method for the given dataset," in Social Informatics (T.-Y. Liu, C. N. Scollon, and W. Zhu, eds.), Lecture Notes in Computer Science, pp. 194-208, Springer International Publishing.

[34] S. Sobolevsky, R. Campari, A. Belyi, and C. Ratti, "General optimization technique for high-quality community detection in complex networks," Physical Review E, vol. 90, p. 012811, July 2014. Publisher: American Physical Society.

[35] M. E. J. Newman, "Modularity and community structure in networks," Proceedings of the National Academy of Sciences, vol. 103, pp. 8577-8582, June 2006.

[36] J. Reichardt and S. Bornholdt, "Statistical mechanics of community detection," Physical Review E, vol. 74, p. 016110, July 2006. Publisher: American Physical Society.

[37] M. E. J. Newman, "Community detection in networks: Modularity optimization and maximum likelihood are equivalent," Physical Review E, vol. 94, p. 052315, Nov. 2016. arXiv: 1606.02319.

[38] A. Amelio and C. Pizzuti, "Is Normalized Mutual Information a Fair Measure for Comparing Community Detection Methods?," in Proceedings of the 2015 IEEE/ACM International Conference on Advances in Social Networks Analysis and Mining 2015, ASONAM '15, (Paris, France), pp. 1584-1585, Association for Computing Machinery, Aug. 2015. 